\title{
Extended Generalized Mean Value Theorem for Functions of One Variable
}

\author{
Phillip Mafuta* \\ Department of Mathematics, University of ZimbabweP.O Box MP167, Mount Pleasant, Harare, Zimbawe
}

\begin{abstract}
In this manuscript, we state and prove a theorem of a similar flavour to the Generalized Mean Value Theorem for functions of one variable. For lack of a better term, we call the theorem "Extended Generalized Mean Value Theorem" and we abbreviate it EGMVT.In addition, we apply Rolle's Theorem to prove the theorem. Also, we deduce some corollaries for Mean Value Theorems. In addition, the EGMVT is verified by use of an example.
\end{abstract}

Key words: Rolle's Theorem; Functions of One Variable; Extended Generalised Mean Value Theorem.

\section{Notation}

Let $f(x)$ be a function of one variable $x$, say. We shall use the notation $f^{\prime}(x)$ to denote the first derivative of the function $f(x)$ with respect to $x$ and $f^{(k)}(x)$ to denote the $k^{\text {th }}$ derivative of a function $f(x)$ with respect to the variable $x$. Moreover, we shall set $f^{(0)}(x)=f(x)$ where necessary, see for example [1,2].

\section{Known Results}

Below we state without proofs well known results in the field of real analysis, see for instance [1,2]. The following three theorems are Rolle's Theorem, Mean Value Theorem and Cauchy's Mean Value Theorem or Generalized Mean Value Theorem respectively. We will use the theorems to prove the results in this paper.

\section{Theorem 1.}

Let $f(x)$ be a continuous function on a closed bounded interval $[a, b]$ and differentiable in an open interval $(a, b)$, with $f(a)=f(b)$. Then $\exists \xi \in(a, b): f^{\prime}(\xi)=0$.

\section{Theorem 2.}

Let $f(x)$ be a continuous function on a closed bounded interval $[a, b]$ and differentiable in an open interval $(a, b)$, with $f(a)=f(b)$. Then $\exists c \in(a, b): f^{\prime}(c)=\frac{f(b)-f(a)}{b-a}$.

\section{Theorem 3.}

Let $f(x)$ and $g(x)$ be continuous functions on a closed bounded interval $[a, b]$ and differentiable in an open interval $(a, b)$, with $g^{\prime}(x) \neq 0, \forall x \in(a, b)$. Then $\exists d \in(a, b): f^{\prime}(d)=\frac{f(b)-f(a)}{g(b)-g(a)}$.

\section{Results}

EGMVT.

Here, we first state and prove what we shall call the Extended Generalized Mean Value Theorem

\section{Theorem 4.}

Let $n \in \mathbb{N}$ and let $f(x), g_{1}(x), g_{2}(x), g_{3}(x), \cdots, g_{n}(x)$ be $n+1$ continuous functions on a closed bounded interval $[a, b]$ and differentiable in an open interval $(a, b)$, with $g_{i}^{\prime}(x) \neq 0 \forall x \in(a, b)$ for $i=$ $1,2,3, \cdots, n$. Then $\exists \xi \in(a, b): f^{\prime}(\xi)=\frac{f(b)-f(a)}{n}\left[\sum_{i=1}^{n} \frac{g_{i}^{\prime}(\xi)}{g_{i}(b)-g_{i}(a)}\right]$.

Proof. Define a function $F(x)$ by:

$F(x)=n(f(x)-f(a))-\sum_{i=1}^{n} \frac{f(b)-f(a)}{g_{i}(b)-g_{i}(a)}\left(g_{i}(x)-g_{i}(a)\right)$.

Since $g^{\prime}(x) \neq 0$, it implies that $g_{i}(b)-g_{i}(a) \neq 0$ for $i=1,2,3, \cdots, n$. Moreover, $n$ is finite, so $F(x)$ is well defined on a closed interval $[a, b]$. Furthermore, $F(a)=F(b)=0$ and $F(x)$ is continuous on a closed bounded interval $[a, b]$, by algebra of continuous functions [2]. In addition, it follows by algebra of differentiable functions [2] that, $F(x)$ is a differentiable function in $(a, b)$. Hence the conditions for Rolle's theorem [1,2] are satisfied, so $\exists \xi \in(b): F^{\prime}(\xi)=0$. This implies that 
$n f^{\prime}(\xi)-\sum_{i=1}^{n} \frac{f(b)-f(a)}{g_{i}(b)-g_{i}(a)} g_{i}^{\prime}(\xi)=0$.

Thus $\exists \xi \in(a, b): f^{\prime}(\xi)=\frac{f(b)-f(a)}{n}\left[\sum_{i=1}^{n} \frac{g_{i}^{\prime}(\xi)}{g_{i}(b)-g_{i}(a)}\right]$

as desired.

Notice that the case $n=1$ yields the Generalized Mean Value Theorem [2]. Below we present an example for $n=2$ to verify the Extended Generalized Mean Theorem.

Example 1:

Consider $f(x)=x+1, g_{1}(x)=x^{2}+4 x-4$ and $g_{2}(x)=x^{2}+3 x$ on an interval [0,3]. Here, all functions are continuous on [0,3] and differentiable in $(0,3)$, with $g_{i}^{\prime}(x) \neq 0 \forall x \in(0,3)$ for $i=1,2$. So the conditions of the theorem are satisfied, hence $\exists \xi \in(0,3): f^{\prime}(\xi)=\frac{f(3)-f(0)}{2}\left[\sum_{i=1}^{2} \frac{g_{i}^{\prime}(\xi)}{g_{i}(3)-g_{i}(0)}\right]$. This implies that $78 \xi=117$ and so $\xi=1.5 \in(0,3)$ as required.

The next result is a corollary to the Extended Generalised Mean Value Theorem.

\section{Corollary 1.}

Let $n \in \mathbb{N}$ and let $f(x), g_{1}(x), g_{2}(x), g_{3}(x), \cdots, g_{n}(x)$ be $n+1$ continuous functions on a closed bounded interval $[a, b]$ and are $k$ times differentiable in an open interval $(a, b)$, with $g_{i}^{k}(x) \neq 0 \forall x \in(a, b)$ for $i=1,2,3, \cdots, n$. Then $\exists \xi \in(a, b): f^{(k)}(\xi)=\frac{f^{(k-1)}(b)-f^{(k-1)}(a)}{n}\left[\sum_{i=1}^{n} \frac{g_{i}^{(k)}(\xi)}{g_{i}^{(k-1)}(b)-g_{i}^{(k-1)}(a)}\right]$.

Proof. Set $\Phi(x)=f^{(k-1)}(x)$ and $\psi_{i}(x)=g_{i}^{(k-1)}(x)$ for $i=1,2,3, \cdots, n$.It follows that

$$
\psi_{i}^{\prime}(x)=g_{i}^{k}(x) \neq 0 .
$$

Moreover both $\Phi(x)$ and $\psi_{i}(x)$ are continuous on $[a, b]$ and differentiablein $(a, b)$. Hence by the EGMVT:

$\exists \xi \in(a, b): \Phi^{\prime}(\xi)=\frac{\Phi(b)-\Phi(a)}{n}\left[\sum_{i=1}^{n} \frac{\psi_{i}^{\prime}(\xi)}{\psi_{i}(b)-\psi_{i}(a)}\right]$.

Hence the result follows.

In example 1 , corollary 1 holds true $\forall \xi \in(0,3)$.

Example 2.Let $f(x)=x^{3}, g_{1}(x)=x^{2}+4 x-4$ and $g_{2}(x)=x^{2}+3 x$ on an interval [0,3]. For this example the Extended Generalized Mean Value Theorem holds true, with $\xi=1.814 \in(0,3)$. Now the three functions are twice differentiable in $(0,3)$ and $g_{i}^{(2)}(x) \neq 0$ for $i=1,2$. So the conditions of the corollary are satisfied, hence $\exists \xi \in(0,3): f^{(2)}(\xi)=\frac{f^{\prime}(b)-f^{\prime}(a)}{2}\left[\sum_{i=1}^{2} \frac{g_{i}^{(2)}(\xi)}{g_{i}^{\prime}(b)-g_{i}^{\prime}(a)}\right]$.

This implies that $6 \xi=9$ and so $\xi=1.5 \in(0,3)$. Therefore the corollary has been verified. In a Similar manner, we can deduce the following corollary to the Mean Value Theorem [2].

\section{Corollary 2.}

Let $k \in \mathbb{N}$ and let $f(x)$ be a continuous functions on a closed bounded interval $[a, b]$ and is $k$ times differentiable in an open interval $(a, b)$. Then $\exists c \in(a, b): f^{(k)}(c)=\frac{f^{(k-1)}(b)-f^{(k-1)}(a)}{b-a}$.

Proof. The proof is similar to that in corollary 1. We simply set $\Phi(x)=f^{(k-1)}(x)$ and apply the Mean Value Theorem [2] on $\Phi(x)$.

\section{Conclusion}

Rolle's Theorem [1,2] has been used as a major tool to prove a theorem of a similar flavor to the Generalized Mean Value Theorem. In fact the case $n=1$ yields the Generalized Mean Value Theorem [2]. Corollaries have been deduced from the Extended Generalized Mean Value Theorem and the Mean Value Theorem [2]. Two examples have been presented to illustrate results.

\section{References}

[1] Dieudonne', J., Foundations of Modern Analysis, Academic Press, (1960)

[2] Rudin, W., Principles of Mathematical Analysis, McGraw-Hill, Inc, (1976) pp: 107-108 